\title{
An Efficient Noncatalytic Protocol for the Synthesis of Trisubstituted Imidazole in Polyethylene Glycol Using Microwaves
}

\author{
Santosh V. Nalage ${ }^{1}$, Mohan B. Kalyankar ${ }^{1}$, Vijay S. Patil ${ }^{1}$, Sidhanath V. Bhosale ${ }^{*}, 1$, Satish U. \\ Deshmukh $^{2}$ and Rajendra P. Pawar ${ }^{*}, 2$

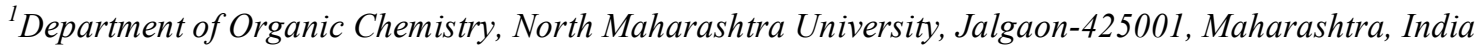 \\ ${ }^{2}$ Department of Chemistry, Deogiri College, Aurangabad-431005, Maharashtra, India
}

\begin{abstract}
An efficient and green procedure for the synthesis of 2, 4, 6-triaryl-1H-imidazole in polyethylene glycol under microwave irradiation in excellent yield has been developed. Polyethylene glycol is non toxic, reusable, inexpensive and easily available.
\end{abstract}

Keywords: PEG, microwave irradiation, trisubstituted imidazole.

Multi substituted imidazole and derivatives are an important class of compounds in the field of pharmaceuticals. They exhibit a wide range of biological activities such as, inhibitors of p38 MAP Kinase [1], B-Raf kinase [2], anti-HIV [3], anticonvulsant [3], HIV-1 protease [4], calcium antagonist and inhibitors of thromboxane A2 synthesase [5] therapeutic agent [6], antihistaminic [7], tranquilizer [8], antimuscarinic [9], antiarthritic [10], cardiotonic [11], HMG CoA reductase(HMGR) [12], and antitumor agents [13]. In recent years substituted imidazoles are substantially used as Ionic liquids [14], a new approach to "green chemistry".

Literature survey reveals that several methods have been developed for the synthesis of 2,4,5-triaryl-1H-imidazoles by three component cyclocondensation of 1,2-diketone, $\alpha$ hydroxy ketone with aldehydes and ammonium acetate, which comprises the use of ionic liquids [15], silica sulphuric acid [16], refluxing in acetic acid [17a-b], alum [18a], sulphanilic acid [18b], $\mathrm{NiCl}_{2} \cdot 6 \mathrm{H}_{2} \mathrm{O}$ [19], $\mathrm{H}_{3} \mathrm{PO}_{4}$ [20], CAN [21], grinding with $\mathrm{I}_{2}$ [22], from $\mathrm{N}$-acylated $\alpha$-amino in presence of triphenyl phosphine followed by coupling with Pd catalyst [23]. Recently, Konwar, et al. reported the synthesis of imidazole using $\mathrm{InCl}_{3} \mathrm{H}_{2} \mathrm{O}$ [24]. Moreover, imidazoles have also been prepared by the reaction of aryl nitriles and $\alpha, \alpha$-dithioaryl nitromethanes [25a],. Microwave assisted synthesis of tri- and tetrasubstituted derivatives was reported by Kidwai et al. But during the reaction they got sticky solid which indicates that, it is not a cleaner approach [25b]. Many of these synthetic methods suffer from one or more drawbacks such as harsh reaction conditions, poor yield, laborious work-up and purifications, prolonged reaction time, etc.

\footnotetext{
*Address correspondence to these authors at the (SVB) Department of Organic Chemistry, North Maharashtra University, Jalgaon-425001, Maharashtra, India; Tel: +91-257-2257433; Fax: +91-257-2258403; E-mail: sidhanath2003@yahoo.co.in and (RPP) Department of Chemistry, Deogiri College, Aurangabad-431005, Maharashtra, India; Tel: +91-240-2487005; Fax: +91-240-2487284; E-mail: rppawar@yahoo.com
}

Moreover, the synthesis of heterocycles in polar solvents led to hazardous complex isolation and recovery procedures. Therefore, the development of new non catalytic method is necessary to overcome their shortcoming, and fulfil mild conditions, efficient and environmentally benign protocol for the synthesis of multisubstituted imidazoles.

Nowadays, interest of chemists increases toward the use of polyethylene glycol as reaction medium as well as catalyst due to its non-toxicity, high efficiency, easy separation and purification, cost effectiveness and reusability. From environmental point of view, it is desirable to use polyethylene glycol as reaction medium and catalyst instead of organic solvents and catalyst, since polyethylene glycol is environmentally benign, cheap, and easily available.

Herein, we report one-step synthesis of imidazoles by the combination of Benzil or Benzoin with aldehyde and ammonium acetate in polyethylene glycol under scientific microwave irradiation. It represents a significant improvement over existing methods of imidazoles synthesis. An advantage of this protocol is the solvent PEG-200 which was recovered by evaporation of water and reused for several times.

In recent years, the use of microwave irradiation in organic synthesis has become an attractive tools [26]. We develop a simple synthetic protocol for imidazoles synthesis from readily available starting materials. The reaction [27] of benzil or benzoin, aldehyde and ammonium acetate in polyethylene glycol under microwave irradiation for 5 to 10 minutes at $900 \mathrm{~W}$ yields imidazoles (Schemes $\mathbf{1}$ and 2). This result prompted us to investigate the imidazole synthesis using different aldehydes (Table 1).

The synthesis of trisubstituted imidazole in different PEG (Scheme 3) was also attempted and no change in the yield was found. Thus the reaction was optimized in PEG-200, 400, 600 (Table 2). In conclusion, a simple one step multicomponent methodology has been developed for the synthesis of 2, 4,5-triaryl-1H-imidazole. 


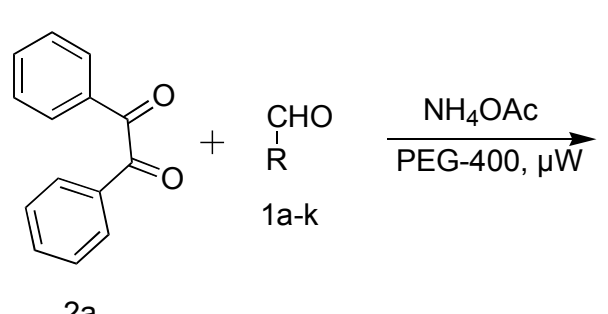

$2 a$

Scheme 1.<smiles></smiles>

$2 b$

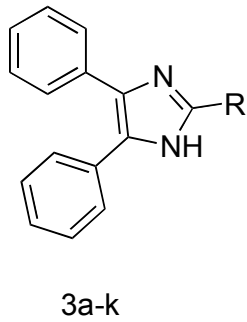

$68-80 \%$<smiles>[R]c1nc(-c2ccccc2)c(-c2ccccc2)[nH]1</smiles>

$3 a-k$

$61-72 \%$

Scheme 2.

Table 1. Synthesis of Trisubstituted Imidazole in Polyethylene Glycol

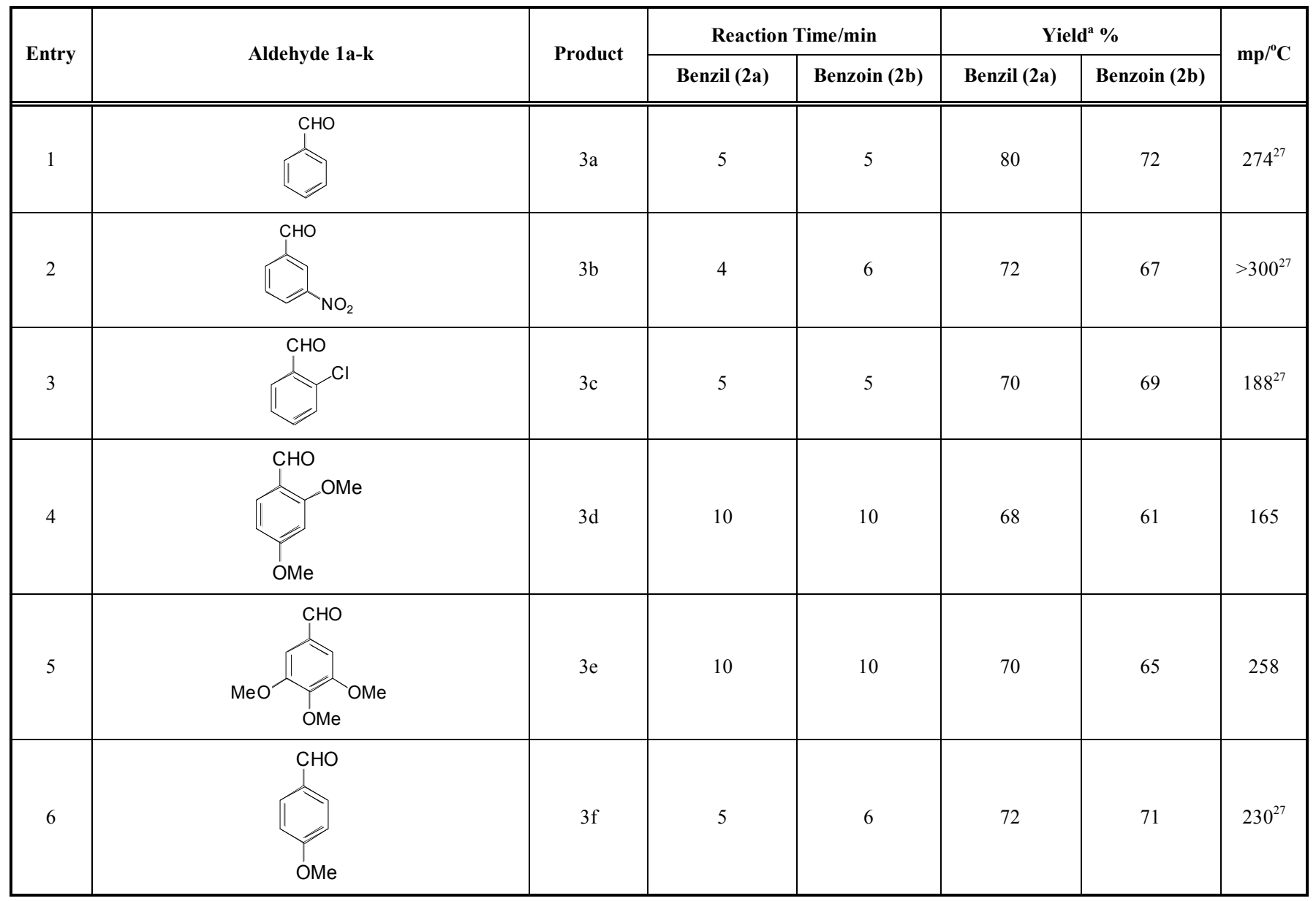




\begin{tabular}{|c|c|c|c|c|c|c|c|}
\hline \multirow{2}{*}{ Entry } & \multirow{2}{*}{ Aldehyde 1a-k } & \multirow{2}{*}{ Product } & \multicolumn{2}{|c|}{ Reaction Time/min } & \multicolumn{2}{|c|}{ Yield $^{a} \%$} & \multirow{2}{*}{$\mathrm{mp} /{ }^{\circ} \mathrm{C}$} \\
\hline & & & Benzil (2a) & Benzoin (2b) & Benzil (2a) & Benzoin (2b) & \\
\hline 8 & & $3 \mathrm{~h}$ & 6 & 7 & 77 & 68 & 218 \\
\hline 9 & & $3 \mathrm{i}$ & 6 & 7 & 68 & 71 & $166^{22}$ \\
\hline 10 & & $3 j$ & 5 & 6 & 70 & 67 & $238^{27}$ \\
\hline 11 & & $3 \mathrm{k}$ & 7 & 7 & 68 & 70 & 235 \\
\hline
\end{tabular}

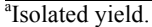

${ }^{\mathrm{b}} \mathrm{All}$ compounds are known in the literature and were characterized by using LC-MS and $\left(400 \mathrm{CDCl}_{3}\right)$ Varian Spectrophotometer.

\section{CONCLUSIONS}

A series of 2,4,5-triaryl-1H-imidazole was synthesized in one step. We demonstrated an efficient protocol for the synthesis of substituted imidazole from benzil/benzoin, aldehydes and ammonium acetate without catalyst in polyethylene glycol. Product isolation via filtration without any hazardous organic solvent claims an environmentally benign protocol for the synthesis of substituted imidazoles.

Table 2. Synthesis of Trisubstituted Imidazole in different PEG

\begin{tabular}{|c|c|c|c|}
\hline Entry & PEG & Reaction Time/min & Yield \% \\
\hline \hline 1 & PEG-200 & 5 & 70 \\
\hline 2 & PEG-400 & 5 & 70 \\
\hline 3 & PEG-600 & 5 & 71 \\
\hline
\end{tabular}

\section{GENERAL PROCEDURE FOR THE PREPARATION OF TRISUBSTITUTED IMIDAZOLE}

All organic solvents used for the synthesis were of analytical grade. Melting points were determined and are uncorrected. IR spectra were recorded on a Shimadzu IR 200 spectrometer. ${ }^{1} \mathrm{H}$ NMR and ${ }^{13} \mathrm{C}$ NMR were recorded on Varian $400 \mathrm{MHz}$ spectrometer using tetramethylsilane as internal reference. Column chromatography was performed on Merck silica gel 60 (70-230 mesh). Thin layer chromatographic separations were performed on Merck Kiesselgel 60 (70-230 mesh).

The microwave reactions were carried out in a Catalyst Systems Model CATA-R $2450 \mathrm{MHz}$ Sciencetific Microwave Oven at $120-140^{\circ} \mathrm{C}$ and $300 \mathrm{~W}$ for the period shown in the Table 1.

A mixture of benzil $(1 \mathrm{mmol})$, aromatic aldehyde $(1 \mathrm{mmol})$, ammonium acetate $(4 \mathrm{mmol})$ and polyethylene glycol $(2 \mathrm{ml})$ was irradiated under microwave oven for appropriate time. The progress of reaction was monitored by TLC (ethyl acetate:methnol 4:1). After completion of reaction, the reaction mixture was cooled to room temperature and poured on $100 \mathrm{ml}$ ice water. The separated solid was filtered and washed with water. The residue was dried, and recrystallized from methanol: water (9:1) mixture.

2,4,5-Triphenyl- $1 \mathrm{H}$-imidazole (3a): Light- yellow solid mp. $271-272^{\circ} \mathrm{C}$ (Lit. [13] $272-273^{\circ} \mathrm{C}$ ). (Found C, 85.02, H, $5.1, \mathrm{~N}, 9.12 \%$. $\mathrm{C} 21 \mathrm{H} 16 \mathrm{~N} 2$ requires $\mathrm{C}, 85.11, \mathrm{H}, 5.44, \mathrm{~N}$, $9.45 \%)$ IR (KBr) cm ${ }^{-1} 3434,2993,2470,16381216 .{ }^{1} \mathrm{H}$ NMR (400 MHz, CDCl3/ DMSO-d6): $\delta \mathrm{H} 8.91$ (s, brs, $1 \mathrm{H}$ ),<smiles>COc1cc(-c2nc(-c3ccccc3)c(-c3ccccc3)[nH]2)ccc1O</smiles>

Scheme 3. 
7.42-8.12 (m 6H ), 7.68-7.71(m, 3H),7.87(m, 6H); ${ }^{13} \mathrm{C}$ NMR (CDCl3/ DMSO-d6): $\delta \mathrm{C} 136.5,129.1,128.5$. 127.2, 122.1 .

\section{ACKNOWLEDGEMENT}

We thank Principal Dr. K. B. Patil, Vice-Chancellor of North Maharashtra University, Jalgaon for providing necessary facilities. SVB is thankful to DST, New Delhi, for the financial support under fast track young scientist program [SR/FTP/CS-82/2007].

\section{REFERENCES}

[1] Lee, J.C.; Laydon, J.T.; McDonnell, P.C.; Gallagher, T.F.; Kumar, S.; Green, D.; McNulty, D.; Blumenthal, M.J.; Keys, J.R.; Vatter, S.W.L.; Strickler, J.E.; McLaughlin, M.M.; Siemens, I.R.; Fisher, S.M.; Livi, G.P.; White, J.R.; Adams, J.L.; Young, P.R. A protein kinase involved in the regulation of inflammatory cytokine biosynthesis. Nature, 1994, 372-739.

[2] Takle, A.K.; Brown, M.J.B.; Davies, S.; Dean, D.K.; Francis, G.; Gaiba, A.; Hird, A.W.; King, F.D.; Lovell, P.J.; Naylor, A.; Reith, A.D.; Steadman, J. G.; Wilson, D.M. The identification of potent, selective and CNS penetrant furan-based inhibitors of B-Raf kinase. Bioorg. Med. Chem. Lett., 2006, 16, 378-81.

[3] Renukadevi, P.; Biradar, J.S.; Hiremath, S.P.; Manjunath, S.Y. Synthesis and antimicrobiasl activity of 1,2-Disubstituted-4-[5'Substituted-2'-phenyl indole-3'-yl) methylene] imidazolin-5(4H)ones. Indian J. Heterocycl. Chem., 1997, 6, 277-280.

[4] Paul, W.B. Heterocyclic HIV-1 protease inhibitors. Org. Lett., 1999, 1(2), 249-252.

[5] Cozzi, P.; Carganico, G.; Fusar, D.; Grossoni, M.; Menichincheri, M.; Pinciroli, V.; Tonani, R.; Vaghi, F.; Salvati, P. Dihydropyridines Imidazoles Thromboxane-A Synthase/antagonists \& inhibitors. J. Med. Chem. 1993, 36, 2964-2972.

[6] Chengzhi, Z.; Sepehr, S.; Edmund, J. M.; Sonja, K.; Jennifer, C. R.; Khalid, D. B.; Ross, D.; Adnan, M. M. 2,4,5-Trisubstituted imidazoles: novel nontoxic modulators of P-glycoprotein mediated, multidrug resistance, Bioorg Med Chem Lett., 2000, 10(23), 26032605.

[7] Robert, A. T.; Charles, F. H.; Caesar, R. S. Studies on imidazole I. 4-methyl imidazole and related compounds. J. Am. Chem. Soc., 1949, 71 (8), 2801-2803.

[8] Shealy, Y.E.; Montogomery, J.A.; Loster, W.R. Antitumor activity of triazenoimidazoles. J. Biochem. Pharmacol., 1962, 11, 674.

[9] Miyachi, H.; Kiyota, H.; Segawa, M. Novel imidazole derivatives with subtype-selective antimuscarinic activity. Bioorg Med. Chem. Lett., 1998, 18, 2163-2168.

[10] Sharpe, T.R.; Cherkovsky, S.C.; Hewes, W.E.; Smith, D.H.; Gregory, W.A.; Haber, S.B.; Leadbetter, M.R.; Whitney, J.G. Preparation, antiarthritic and analgesic activity of 4,5-diaryl-2(substituted thio)-1H-imidazoles and their sulfoxides and sulfones. J. Med. Chem., 1985, 28, 1188-1194.

[11] Ila Sircar, Bradley L. Duell, James A. Bristol, Ronald E. Weishaar, Dale B. Evans Cardiotonic agents. 5. 1,2-Dihydro-5-[4- $(1 \mathrm{H}-$ imidazol-1-yl)phenyl]-6-methyl-2- oxo-3-pyridinecarbonitriles and related compounds. Synthesis and inotropic activity. J. Med. Chem., 1987, 30, 1023-1029.

[12] Chuen, C.; Bailey, E. J.; David, H.; David, F. H.; Julie, L. H.; Graham, G. A. I.; Paul, S. J.; Suzanne, E. K.; Barrie, E. K. Inhibitors of cholesterol biosynthesis. 1. 3,5-Dihydroxy-7-(Nimidazolyl)-6-heptenoates and -heptanoates, a novel series of 3hydroxy-3-methylglutarate-CoA reductase inhibitors. J. Med. Chem., 1996, 36, 3646-3657.

[13] Wang, L.; Woods, K.W.; Li, Q.; Barr, K.J.; McCroskey, R.W.; Hannick, S. M.; Gherke, L.; Credo, R.B.; Hui, Y.H.; Marsh, K.;
Warner, R.; Lee, J.Y.; Zielinsky-Mozng, N.; Frost, D.; Rosenberg, S.H.; Sham, H.L. Potent, Orally active heterocycle-based combretastatin A-4 analogues: synthesis, structure-activity relationship, pharmacokinetics, and in vivo antitumor activity evaluation J. Med. Chem., 2002, 45, 1697-1711.

[14] (a) Dupont, J.; de Souza, R.F.; Suarez, P.A.Z. Ionic liquid (molten salt) phase organometallic catalysis. Chem. Rev., 2002, 102, 3667; (b) Nara, S.J.; Naik, P.U.; Harjani, J.R.; Salunkhe, M.M. Potential of ionic in greener methodologies involving biocatalysis and other synthetically important transformations). Indian J. Chem., 2001, 45B, 2257; (c) Chowdhury, S.; Mohan, R.S.; Scott, J.L. Reactivity of ionic liquids. Tetrahedron, 2007, 63, 2363.

[15] Siddiqui, S.A.; Narkhede, U.C.; Palimkar, S.S.; Daniel, T.; Lahoti, R.J.; Srinivasan, K.V. Room temperature ionic liquid promoted improved and rapid synthesis of 2,4,5-triaryl imidazoles from aryl aldehydes and 1,2-diketones or $\alpha$-hydroxyketone. Tetrahedron, 2005, 61, 3539-3546.

[16] Shaabani, A.; Rahmati, A. Silica sulfuric acid as an efficient and recoverable catalyst for the synthesis of trisubstituted imidazoles. $J$. Mol. Catal. A Chem., 2006, 249, 246-248.

[17] (a) Sarshar, S.; Siev, D.; Mjalli, M.M. Imidazole libraries on solid support. Tetrahedron Lett; 1996, 37, 835-838; (b) Gallagher, T.F.; Seibel, G.L.; Kassis, S.; Laydon, J.T.; Blumenthal, M.J.; Lee, J.C.; Lee, D.; Boehm, J. C.; Fier-Thompson, S.M.; Abt, J.W.; Soreson, M.E.; Smietana, J.M.; Hall, R. F.; Garigipati, R.S.; Bender, P.E.; Erhard, K.F.; Krog, A.J.; Hofmann, G.A.; Sheldrake, P.L.; McDonnell, P.C.; Kumar, S.; Young, P.R.; Adams, J.L. Regulation of stress-induced cytokine production by pyridinylimidazoles; inhibition of CSBP kinase. Bioorg. Med. Chem. 1997, 5, 49.

[18] (a) Mohammadi, A. A.; Mivechi, M.; Kefayati, H. ChemInform abstract: potassium aluminum sulfate (Alum): an efficient catalyst for the one-pot synthesis of trisubstituted imidazoles. Monatsh Chem., 2008, 139, 935-937; (b) Mohammed, A.F.; Kokare, N.D.; Sangshetti, J.N.; Shinde, D.B. Sulphanilic acid catalyzed facile one-pot synthesis of 2,4,5-triarylimidazoles. from Benzil/Benzoin and aromatic aldehydes. J. Korean Chem. Soc. 2007, 51, 418-422.

[19] Heravi, M.M.; Bakhtiari, K.; Oskooie, H.A.; Taheri, S. Synthesis of 2,4,5-triaryl-imidazoles catalyzed by $\mathrm{NiCl}_{2} \cdot 6 \mathrm{H}_{2} \mathrm{O}$ under heterogeneous system. J. Mol.Catal. A Chem., 2007, 263, 279.

[20] Liu, J.; Chem, J.; Zhao, J.; Zhao, Y.; Li, L.; Zhang, H. A modified procedure for the synthesis of 1-arylimidazoles. Synthesis, 2003, 17, 2661-2266.

[21] Sangshetti, J.N.; Kokare, N.D.; Kotharkara, S.A.; Shinde, D.B. A new series of 4-substituted 3H-1,2,3,5-oxathiadiazole 2-oxides bearing 1,2. J. Chem. Sci., 2008, 120(5), 463-467.

[22] Parveen, A.; Ahmed, M.D.; Rafi, S.K.; Shaikh, K.A.; Deshmukh, S.P.; Pawar, R.P. Efficient synthesis of 2,4,5-triaryl substituted imidazoles under solvent free conditions at room temperature. Arkivoc, 2007, 12-18.

[23] Zhong, Y.L.; Lee, J.; Reamer, R. A.; Askin, D. Divergent and Regioselective synthesis of $1,2,4-$ and 1,2,5-trisubstituted imidazoles. J. Org. Chem., 2008, 73(17), 6816- 6823.

[24] Sharma, S.D.; Hazarika, P.; Konwar, D. An efficient and one-pot synthesis of 2,4,5-trisubstituted and 1,2,4,5-tetrasubstituted imidazoles catalyzed by $\mathrm{InCl}_{3} \cdot 3 \mathrm{H}_{2} \mathrm{O}$. Tetrahedron Lett., 2008, 49 , 2216-2220.

[25] (a) Hayes, J.F.; Mitchell, M.B.; Wicks, C. Heterocycles, 1994, 38 , 575; (b) Kidwai, M.; Saxena, S.; Rastogi, R.; Rastogi, S. An efficient synthesis of 2,4,5-trisubstituted and 1,2,4,5tetrasubstituted-1H-imidazoles. Bull. Korean Chem. Soc., 2005, 26(12), 2051-2053.

[26] Lidsrom, P.; Tierney, J.; Wathey, B.; Westman, J. Microwave assisted organic synthesis-a review. Tetrahedron, 2001, 57, 9225.

[27] Xia, M.; Lu, Y. A novel neutral ionic liquid-catalyzed solvent-free synthesis of 2,4,5-trisubstituted imidazoles under microwave irradiation. J. Mol. Catal. A Chem., 2007, 265, 205-208. 